\title{
A Highly Sensitive Dopamine Sensor Based on Graphene Quantum Dots Modified Glassy Carbon Electrode
}

\author{
Shengbiao Zheng ${ }^{l}$, Rong Huang ${ }^{l}$, Xiaoqing $\mathrm{Ma}^{l}$, Jing Tang ${ }^{1 *}$, Zirong $\mathrm{Li}^{l}$, \\ Xuchuan Wang ${ }^{I}$, Jumeng Wei ${ }^{1}$, Jianfei Wang, ${ }^{2, *}$ \\ ${ }^{1}$ College of Chemistry and Material Engineering, Anhui Science and Technology University, 233100, \\ Fengyang, Anhui Province, China \\ ${ }^{2}$ College of Resource and Environment, Anhui Science and Technology University, 233100, \\ Fengyang, Anhui Province, China \\ *E-mail: zhengtang102@163.com (Tang Jing), wangjf@ahstu.edu.cn (Wang Jianfei)
}

doi: $10.20964 / 2018.06 .19$

Received: 26 January 2018 / Accepted: 18 March 2018 / Published: 10 May 2018

\begin{abstract}
A graphene quantum dots (GQDs) modified glassy carbon electrode (GCE), namely GQDs/GCE was prepared by electrodeposition method, which was characterized by chronocoulometry and electrochemical impedance spectra. The electrochemical behavior of the prepared modified electrode towards the catalytic oxidation of dopamine (DA) in $\mathrm{pH} 7.0$ phosphate buffer solution was investigated by cyclic voltammetry and differential pulse voltammetry. It was demonstrated that the modified electrode presented good electrocatalytic activity and had favorable electrochemical response towards DA without the interference of ascorbic acid. The probable mechanism on the modified electrode surface was proposed. Under optimum conditions, the dependence of current vs. concentration was linear from 0.4 to $100 \mu \mathrm{M}$ with a regression coefficient of 0.997 , and the detection limit of DA was 50 $\mathrm{nM}(S / N=3)$. Analytical application of GQDs/GCE was successfully tested in the determination of DA in real samples.
\end{abstract}

Keywords: graphene quantum dots (GQDs); dopamine; modified electrode; ascorbic acid

\section{FULL TEXT}

(C) 2018 The Authors. Published by ESG (www.electrochemsci.org). This article is an open access article distributed under the terms and conditions of the Creative Commons Attribution license (http://creativecommons.org/licenses/by/4.0/). 